\title{
Zero misorientation interfaces in graphene
}

\author{
M. A. Rozhkov ${ }^{1}$, N. D. Abramenko ${ }^{1}$, A. L. Kolesnikova ${ }^{1,2}$, A. E. Romanov ${ }^{\dagger, 1,3}$ \\ †alexey.romanov@niuitmo.ru
}

\author{
${ }^{1}$ ITMO University (National Research University ITMO), 49 Kronverksky pr., St. Petersburg, 197101, Russia \\ ${ }^{2}$ Institute for Problems in Mechanical Engineering, RAS, 61 Bolshoj pr., Vas. Ostrov, St. Petersburg, 199178, Russia \\ ${ }^{3}$ Ioffe Physical-Technical Institute, RAS, 26 Polytechnicheskaya st., St. Petersburg, 194021, Russia
}

\begin{abstract}
This article presents the results on the modeling of straight-line interfaces that induce no misorientation of adjacent regions in graphene: zero misorientation interfaces (ZMIs). The interfaces in the hexagonal graphene lattice are represented as ensembles of disclinated carbon rings with broken rotational symmetry of the sixth order. The basic elements of such ensembles are structural units - complexes of disclinated rings with zero disclination charge. Using molecular dynamics simulation, the energies and atomic densities for ZMIs are found. Calculations demonstrate that atomic densities in ZMIs are lower than the atomic density in defect-free graphene. No direct correlation has been revealed between the atomic density and the interface energy. It is assumed, that the elastic field caused by ZMI defect structure contributes significantly to the energy of interface. Low-energy ZMIs possess linear energies not exceeding $\sim 0.6-0.8 \mathrm{eV} / \AA$, that is comparable to the energies of the grain boundaries, i.e. boundaries with misorientation, in graphene. Based on a mesoscopic approach operating with disclination schemes, in which defective carbon rings are replaced by disclinations, strain maps are plotted, and energies are found for two selected low-energy ZMIs. It is demonstrated that, at the distance of ZMI half-period from interface line, strains decrease to values of $\sim 0.05$. The energies of low-energy ZMIs calculated within the framework of two approaches: atomistic and mesoscopic, although differ numerically, coincide by the order of magnitude.
\end{abstract}

Keywords: graphene, interfaces, zero misorientation, disclinations, molecular dynamics.

\section{Introduction}

Graphene is a two-dimensional carbon crystal that has promising properties for practical applications: it has high electrical conductivity [1] and thermal conductivity [2], as well as a unique set of mechanical characteristics [3]. Its successful synthesis [4] has caused an active increase in the interest of scientists and engineers in the study of graphene and two-dimensional crystals in general.

In graphene, as in three-dimensional crystals, crystallattice defects are observed, for details, see reviews $[5,6]$. Defects in graphene can be classified according to the dimension of the region of their localization into two-dimensional (pores and inclusions), one-dimensional (interfaces), and point-like (vacancies, interstitial and impurity atoms, dislocations and disclinations) ones.

The influence of defects on the characteristics of graphene crystals turns out to be significant; for example, it is known that the presence of interfaces in graphene can increase thermal conductivity [7] and (or) electrical conductivity [8] and reduce strength $[6,9]$.

In graphene, interfaces, including grain boundaries, are represented as localized ensembles of carbon rings with broken hexagonal symmetry, i.e. chains of five-member, seven-member and other non-six-member rings. The width of the interfaces is much less than their length and is comparable to the lattice parameter. Interfaces in graphene can be divided into grain boundaries and zero misorientation interfaces
(ZMIs). Defects of the last type are observed experimentally $[8,10]$, and are also investigated theoretically $[7,11,12]$.

In this work, we investigate the structure, energy, and elastic distortions of ZMIs in graphene using two theoretical approaches: an analytical mesoscopic approach within the framework of the continuum disclination theory and atomistic modeling by the molecular dynamics (MD) method based on the chosen interatomic interaction potential.

\section{Nomenclature of interfaces in flat 2D crystals}

Interfaces in two-dimensional crystals arise when two adjusting single-crystal regions are rotated or displaced relative to each other by angles or displacements that do not correspond to the rotational or translational symmetry of the initial two-dimensional crystal lattice, see Fig. 1.

Interfaces in two-dimensional crystals can be classified according to their geometric and structural features.

Geometrically, the interface can be straight-line, curved, and faceted as it is schematically shown in Fig. 2.

By structural features, interfaces are subdivided into:

a) Grain boundaries, when the lattices of the two regions (grains) of the crystal are rotated relative to each other (Fig. $3 \mathrm{a}$ ), and interfaces without adjusting region misorientation (Fig. 3b);

b) Symmetric (Fig. 3 c), when the interface line is the axis of symmetry of the crystal lattices of the adjoining regions, and nonsymmetric (Fig. 3 d) otherwise; 
c) Interfaces between regions, the crystal lattices of which are not shifted (interfaces without a shift, Fig. $3 \mathrm{e}$ ) or shifted (interfaces with a shift, Fig. $3 \mathrm{f}$ ) relative to each other along the interface line.

In graphene, interface can be also a linear structure in which the atomic bond hybridization inherent to the original material is disrupted. For example, $s p^{1}$ or $s p^{3}$ hybridizations can be observed in the interface region, instead of the initial $s p^{2}$ hybridization of the electron shells of carbon atoms [11]. In addition, straight-line ZMIs can go along either "zigzag" or "armchair" directions in graphene lattice; the examples of such interfaces are given below in Section 4.1.

\section{Background}

\subsection{Molecular dynamics modeling technique in application to graphene with defects}

MD is a method of atomistic modeling in which the laws of classical mechanics are used to find the positions of interacting atoms. In the MD method, the potential energy of interaction between atoms is selected for the corresponding simulation problem. In particular, the following interatomic interaction potentials are mainly used to simulate graphene:
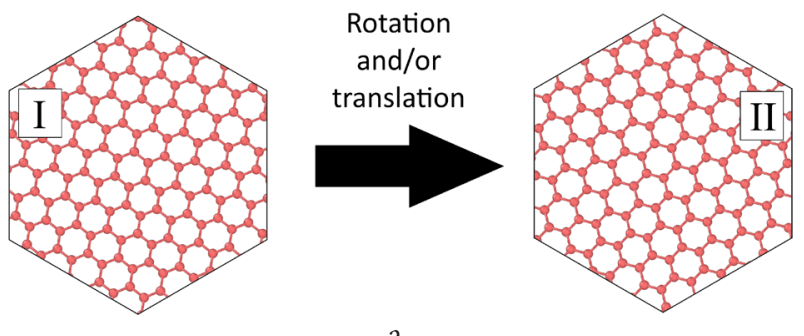

a

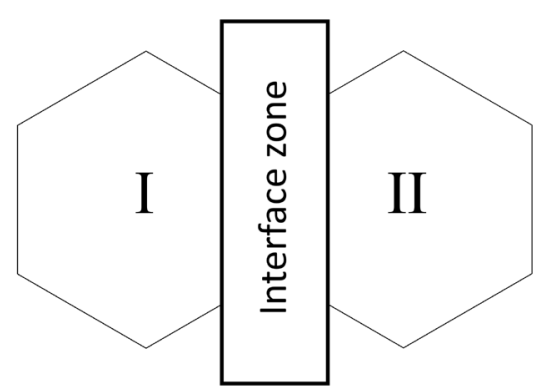

$\mathrm{b}$

Fig. 1. (Color online) Procedure for forming an interface in graphene. Transformation of lattice I into lattice II. I — initial crystal, II - crystal after transformation (a). An interface in graphene formed by joining crystal II and I (b).
AIREBO [13], Tersoff [14], and ReaxFF [15], which take into account the pair interactions of neighboring atoms as well as long-range collective interatomic interactions. These potentials also include parameters responsible for changing the angles between the covalent atomic bonds of carbon atoms.

The choice of one of the three potentials indicated above is determined by the modeling tasks and the possibility of calibrating the potential parameters when comparing the simulation results with the experimental data. For example, if the modeling task is to find the elastic constants of the graphene, then neither Tersoff [16] nor ReaxFF [17] give the values verified by experiment [3]. On the contrary, the AIREBO potential performs well for solving such a problem when analyzing the structure of the graphene subjected deformation [18].

The AIREBO potential was used to simulate graphene with defects [19-22]. In Ref. [20], with the help of the
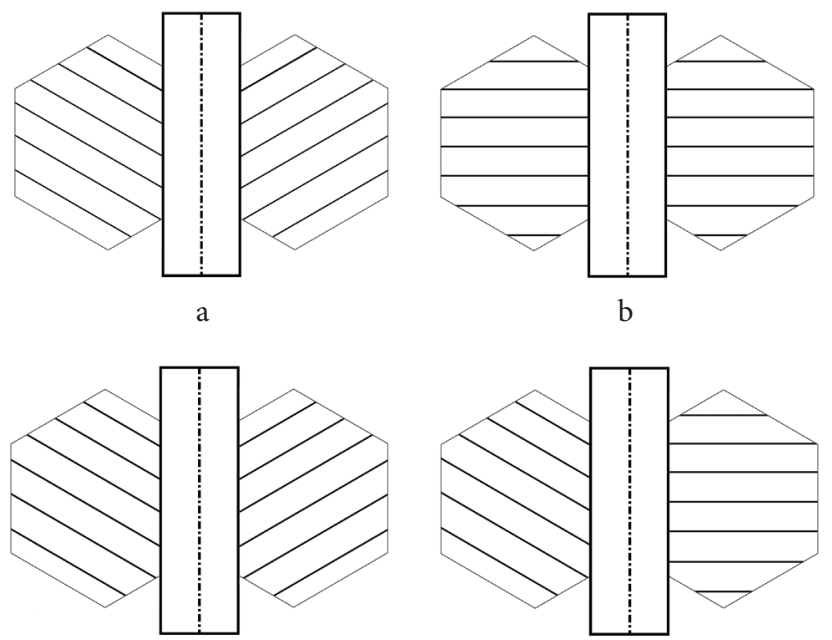

c

d

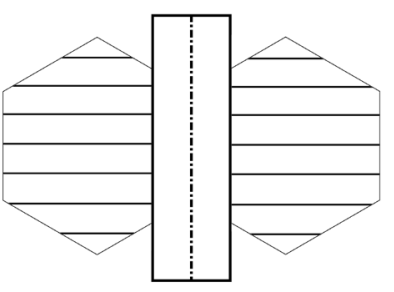

e

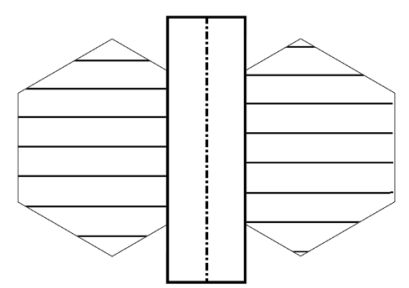

$\mathrm{f}$

Fig. 3. Straight-line interface in graphene: misorientation interface (a); zero misorientation interface (ZMI) (b); symmetric interface (c); nonsymmetric interface $(d)$; interfaces without a shift (e); interface with a shift (f).
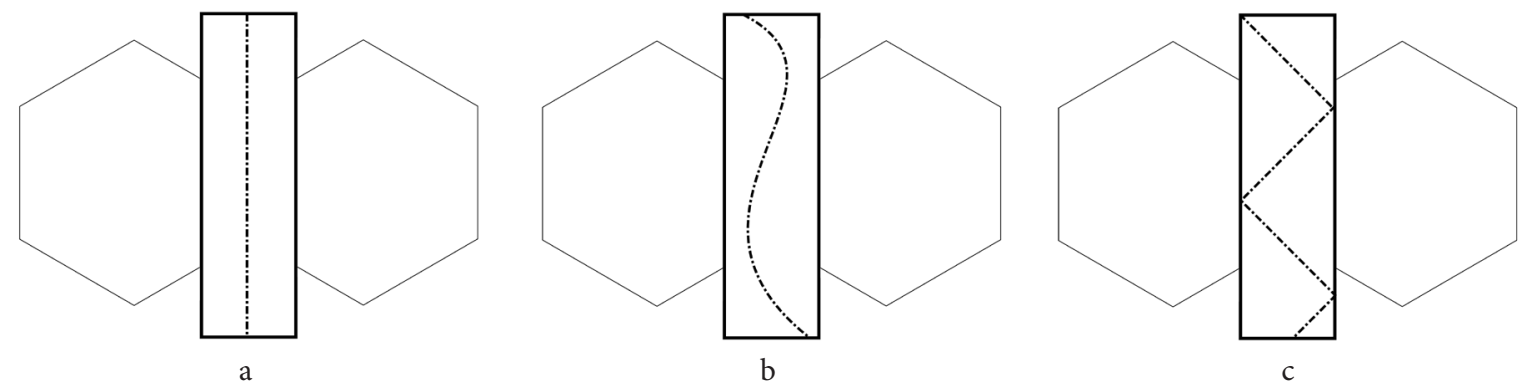

Fig. 2. Straight-line (a), curved (b) and faceted (c) interfaces in 2D crystals. 
AIREBO potential, a study of interfaces in graphene was made and the interfaces structures and energies per unit length (linear energies) were found. In Ref. [19], the simulation of pseudo-graphene crystals - graphene crystals with dense periodic distributions of disclinations - was carried out. In Ref. [21], the thermal conductivity of the interface with zero misorientation was studied. The authors of Ref. [22] simulated tensile fracture of a graphene crystal containing divacancy. In Ref. [23], the effect of the Stone-Wales defects on structural stability of graphene crystal was investigated, and in Ref. [24] the new interatomic potential for description of mechanical properties of graphene was introduced and the comparison with AIREBO potential was shown.

In this work, the AIREBO potential is used in the LAMMPS software package [25] to simulate ZMIs at zero temperature with energy minimization using the Polak-Ribière conjugate gradient algorithm [26]. The results obtained are visualized in the OVITO software package [27].

\subsection{Defects in graphene in relation to disclinations: mesoscopic approach}

It is natural to describe defective carbon rings with broken axial symmetry within the disclination approach [28]. Thus, the five-member carbon ring is the core of a positive wedge disclination with strength (charge) $\omega=+\pi / 3$, the sevenmember ring is the core of a negative wedge disclination with the strength $\omega=-\pi / 3$, the eight-member ring is the core of $\mathrm{a}-2 \pi / 3$-charged wedge disclination, etc. [19].

The continual disclination theory makes it possible to calculate analytically the long-range elastic fields and the excess energy in the crystal, in our case graphene, with individual disclinations and with interfaces consisting of disclinations, when the energy of a perfect crystal is taken as zero energy. To calculate the energy of the interface or disclination network [19], the self-energy of disclinations and the energy of interaction between them are summed.

The advantage of the disclination approach is the understanding of which disclination configuration can be used to compose one or another graphene structure, see, for example, $[6,19,20,29,30]$. In particular, the period of the disclination grid in pseudo-graphene crystals should contain the quadrupoles and the other multipoles of the wedge disclinations with zero charge and moment [19], and the period of the interface can include only structural elements with zero disclination charge $[20,29]$.

In the present work, in the framework of mesoscopic disclination approach, we calculate the strains and the energies of two low-energy ZMIs, and then compare the latest with ZMI energies found in MD simulations.

\section{Properties of zero misorientation interfaces (ZMIs) in graphene}

\subsection{Results of MD modeling of ZMIs}

The construction of structural models of ZMIs was implemented in two stages in MATLAB software package [31]. First, a structural unit (SU) was selected, which was multiplied by translation to create a chain of defective carbon rings along the interface line. Each SU was a disclination configuration with zero total charge: $\sum \omega_{i}=0$. For more details on structural units, see $[19,29]$. Then, sheets of single-crystal graphene were attached to the SU chain, which becomes the interface core, from both sides. As a result, the initial file of the structure with the coordinates of carbon atoms was generated for LAMMPS software package, in which the final configuration of atoms was then found by the MD method.

In this work, we investigated symmetric straightline ZMIs without breaking the $s p^{2}$ hybridization of the electron shells of carbon atoms with the densest packing of disclination defects. The structures of the studied ZMIs are shown in Fig. 4. The used nomenclature of the straight-line ZMIs, which is shown in Fig. 4, corresponds to the content of defective (disclinated) rings in one structural unit, and the letter at the end of the name corresponds to a variant of the interface with the same composition of disclinations in structural units. For the convenience of visual analysis of the considered configuration, disclinated rings with different disclination charges (in other words, rings with different numbers of atoms) were marked with different colors. There is a corresponding disclination diagram next to each ZMI. The angle $\theta$ is responsible for the orientation of the graphene crystal upon contact with the ZMI: $\theta=0^{\circ}$ corresponds to the "armchair" orientation, $\theta=60^{\circ}-$ to the "zigzag" orientation. As a result, for example, ZMI 5-8-5 A1 goes along the "armchair" direction, but ZMI 5-8-5 A2 goes along "zigzag" direction.

After optimizing the structure, i.e. carrying out the relaxation procedure in LAMMPS software package, the linear energy of the ZMIs were calculated. For this, the energies of the crystal region without and with the interface were compared. For more details on the choice of boundary conditions and the size of the analyzed region in MD simulation, see [32].

The energies of the ZMIs studied by the MD method are given in the diagram, see Fig. 5. As follows from the analysis of the results obtained, ZMIs 5-7D, 5-8-5 A1 and 5-8-5D demonstrate the lowest energies, and ZMIs 10-44B, 9-4-5 and $5-7 \mathrm{~B}$ possess the highest energies. For such ZMIs, the atomic density was found in the interface region. For this, in a crystal with ZMI, after relaxation, a ZMI region of an arbitrary length was considered, including at least three structural units, and the transverse boundaries of the region were specified at the ends of defective ZMI rings. The results obtained are shown in Fig. 6, where the atomic density of the boundaries is normalized to the atomic density of defect-free graphene.

According to the data shown in Figs. 5 and 6, no correlation is observed between the energy of the ZMI and its atomic density.

\subsection{Mesoscopic description of ZMIs in graphene}

As noted above, the properties of the ZMIs shown in Fig. 4 can be analyzed within the mesoscopic approach. For this, we use the disclination description of ZMIs and apply the methods and formulas of the continual disclination theory [28]. For example, by selecting the screened disclination configurations, it is possible to depict maps of elastic fields near ZMIs. 


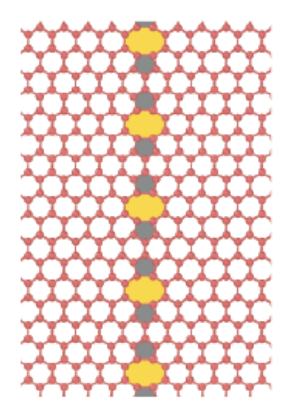

5-8-5 A1

$\theta=0^{\circ}$

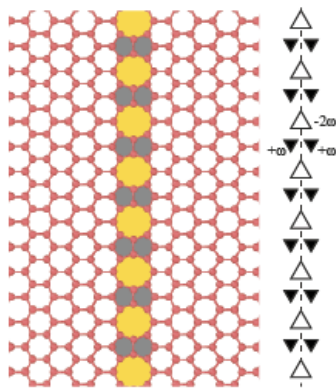

5-8-5 D

$\theta=60^{\circ}$

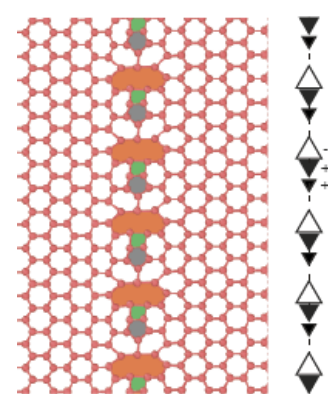

9-4-5

$\theta=60^{\circ}$

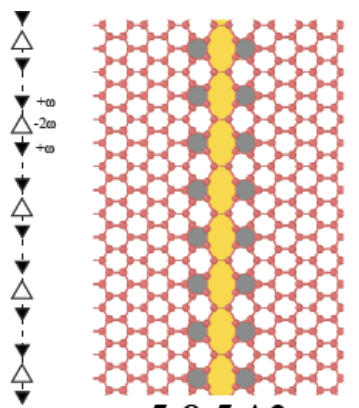

5-8-5 A2

$\theta=60^{\circ}$

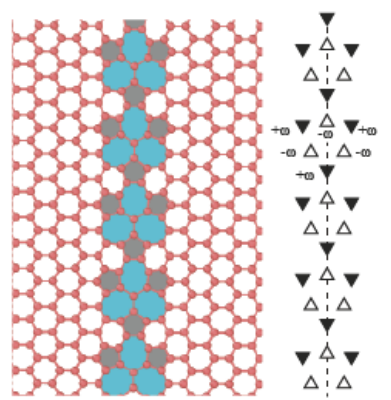

5-7 B

$\theta=60^{\circ}$

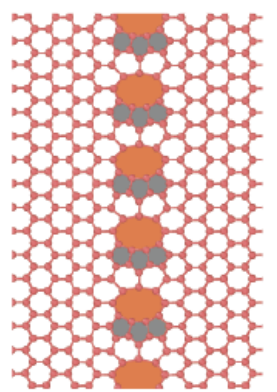

9-555 C

$\theta=60^{\circ}$
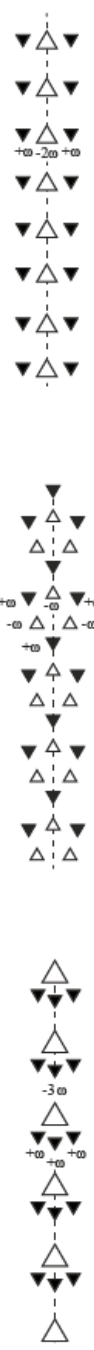

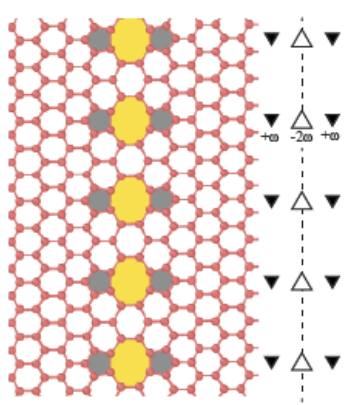

5-8-5 A3

$\theta=60^{\circ}$

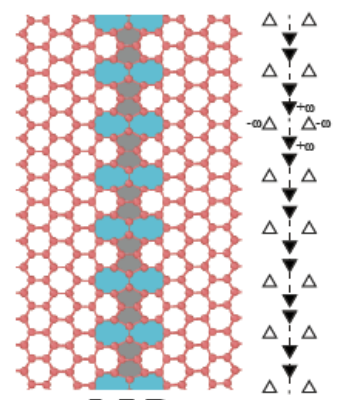

5-7 D

$\theta=60^{\circ}$

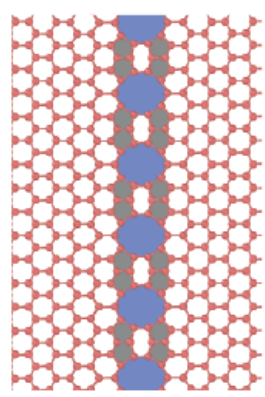

10-555C

$\theta=60^{\circ}$

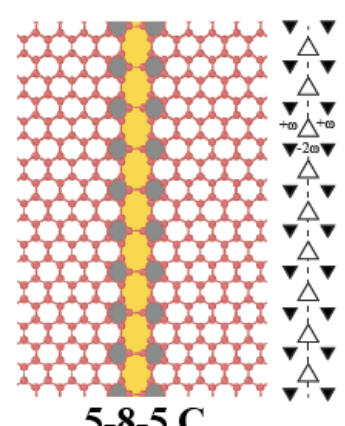

5-8-5 C

$\theta=0^{\circ}$
7-4-7

$\theta=0^{\circ}$

Fig. 4. (Color online) Zero misorientation interfaces (ZMIs) in graphene and their corresponding disclination schemes. Minimum disclination strength modulo $\omega=\pi / 3$. The angle $\theta$ describes the orientation of the crystal regions adjacent to the ZMI. Defective rings are marked with the following colors: green -4 -member ring, gray -5 -member ring, blue -7 -member ring, yellow -8 -member ring, orange -9 -member ring, blue -10 -member ring.

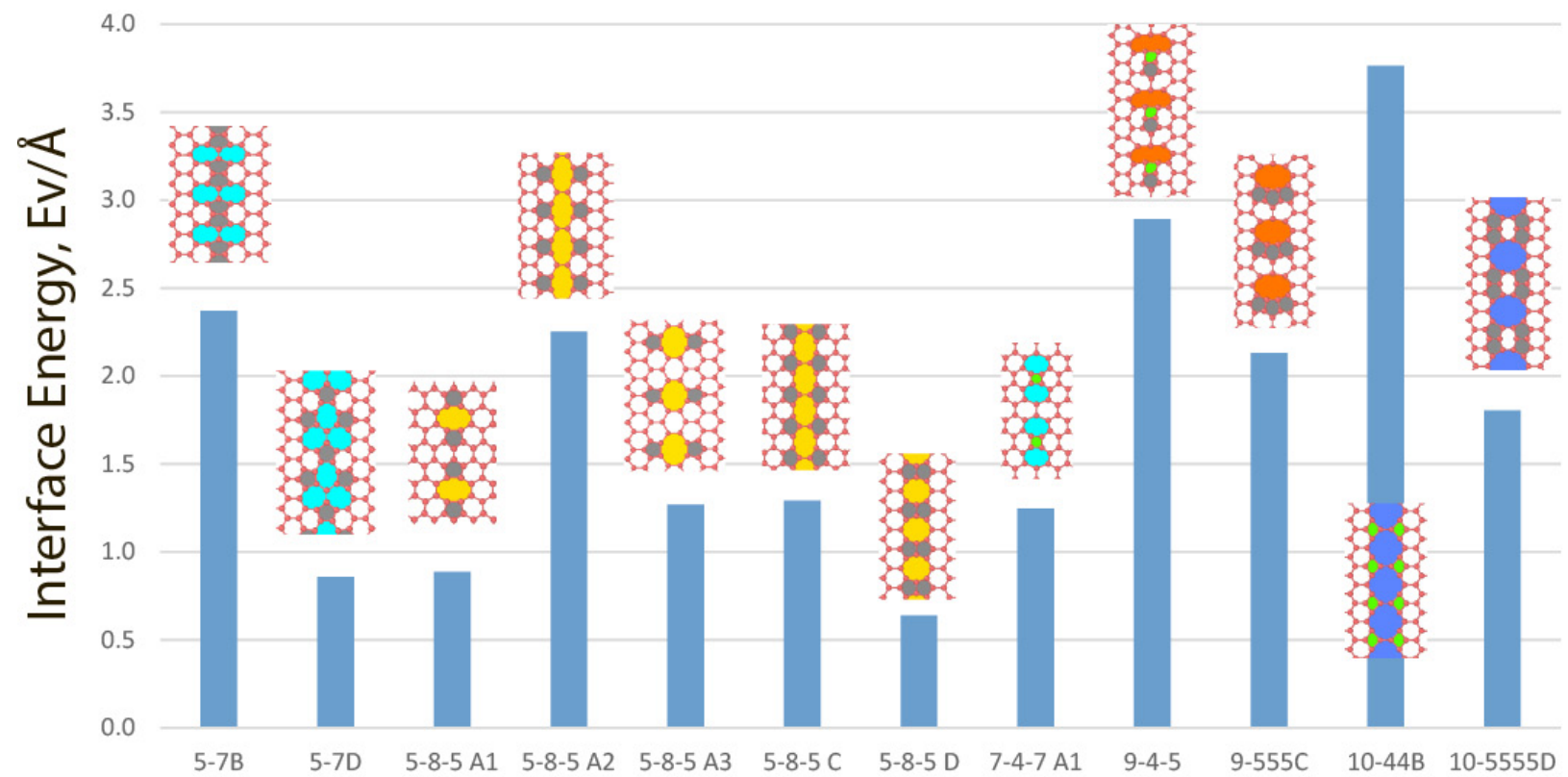

Fig. 5. (Color online) Linear energies of ZMIs in graphene. The insets show the structures of the ZMIs. 
Figs. 7 and 8 show strain maps generated by disclination configurations simulating low-energy ZMIs 5-8-5 D and 5-8-5 A1. For convenience of presentation, the period of ZMI is taken as a unit of length of the region. It follows from the results obtained that the strain components rapidly decay with distance from the ZMI line. At the distance of the halfperiod ZMI, the strains decrease to values of $\sim 0.05$.

Formulas of the disclination theory give possibility to calculate the energies of the considered screened disclination configurations $[19,28]$, and thereby to find the linear energy of ZMIs within the framework of the mesoscopic approach.
In this work, the energies of ZMIs 5-8-5 D and 5-8-5 A1 were found in two approximations: taking into account either a separate disclination quadrupole included in the SU of one interface period, or the interacting quadrupoles of two interface periods. The results of analytical calculations are summarized in Table 1, where, for comparison, the energies of ZMIs calculated using the MD method are shown. The shear modulus $G$ and Poisson's ratio $v$ used in the expression for the quadrupole energy $[19,28]$, were found by the MD simulation with AIREBO potential of defect-free graphene and amounted $G=367 \mathrm{GPa}$ and $v=0.367$, respectively.

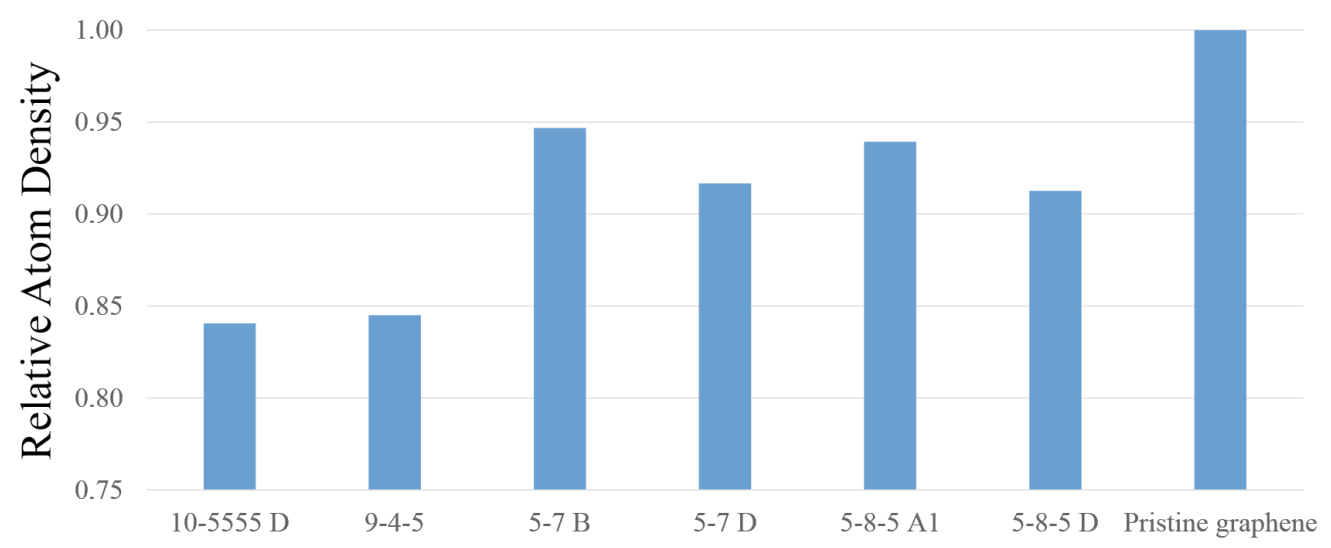

Fig. 6. Diagram of the relative densities of the atoms in the ZMI regions versus the atomic density of defect-free graphene. The density of atoms in defect-free graphene is taken as a unit. The first three ZMIs 10-5555 D, 9-4-5, 5-7 B have the highest linear energy; the next three ZMIs 5-7 D, 5-8-5 A1, 5-8-5 D have the lowest linear energy.

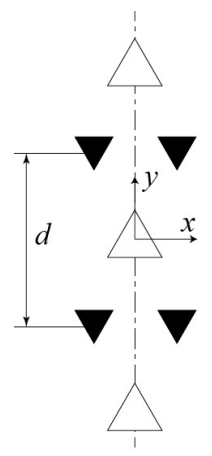

a

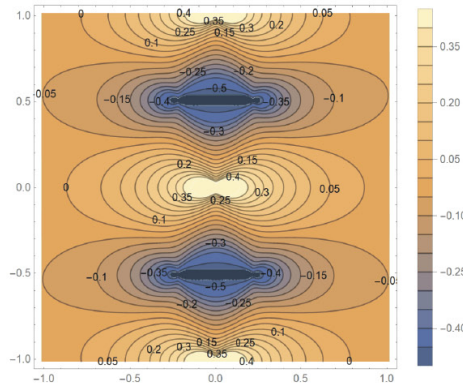

$\varepsilon_{x x}$

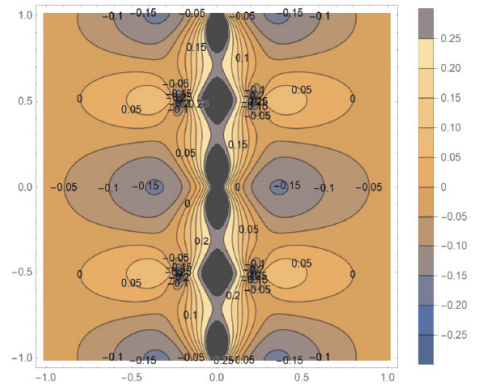

$\varepsilon_{\text {yy }}$

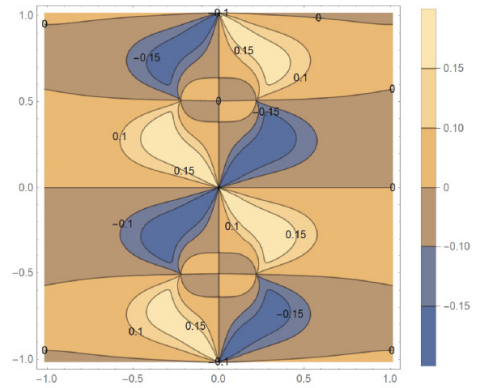

$\varepsilon_{\mathrm{xy}}$

Fig. 7. (Color online) Disclination scheme (a) and strain maps (b-d) for ZMI 5-8-5 D. The length of the period ZMI $d$ is taken as the unit of the linear scale.

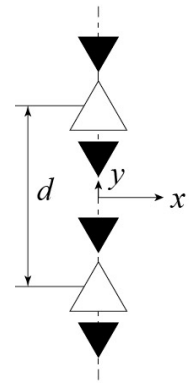

a

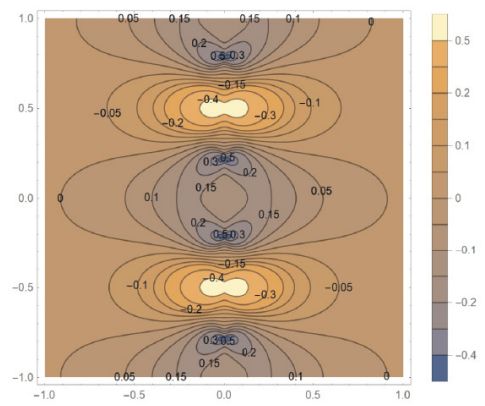

$\varepsilon_{x x}$

$\mathrm{b}$

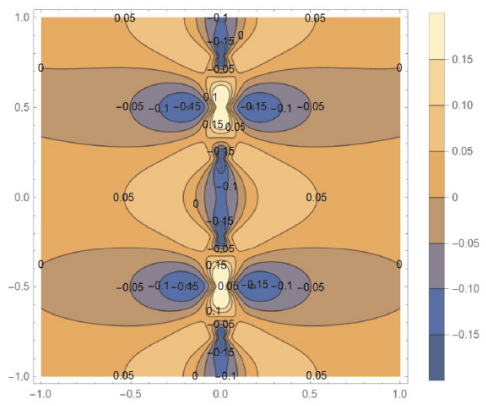

$\varepsilon_{\mathrm{yy}}$

$c$

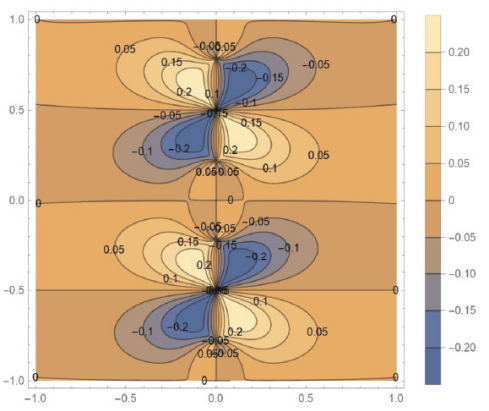

$\varepsilon_{\mathrm{xy}}$

$\mathrm{d}$

Fig. 8. (Color online) Disclination scheme (a) and strain maps (b-d) for ZMI 5-8-5 A1. The length of the period ZMI $d$ is taken as the unit of the linear scale. 
Table 1. Comparison of the linear energies calculated in the framework of atomistic and mesoscopic approaches for low-energy ZMIs.

\begin{tabular}{|c|c|c|c|}
\hline \multirow{2}{*}{ Interface } & \multirow{2}{*}{ Energy calculated by $\mathrm{MD}, \mathrm{eV} / \AA$} & \multicolumn{2}{|c|}{ Analytically calculated energy, eV/Å } \\
\hline & & $\begin{array}{l}\text { Taking into account the disclination } \\
\text { configuration of one ZMI period }\end{array}$ & $\begin{array}{l}\text { Taking into account the disclination } \\
\text { configuration of two ZMI period }\end{array}$ \\
\hline 5-8-5 D & 0.626 & 1.032 & 0.757 \\
\hline 5-8-5 A1 & 0.899 & 0.592 & 0.584 \\
\hline
\end{tabular}

\section{Discussion}

The 5-8-5 D interface was previously considered in Ref. [11] along with two other ZMIs, for which the initial $s p^{2}$ hybridization of graphene is disrupted. The researchers concluded that ZMIs without disturbing hybridization could increase the electronic thermal conductivity of a material. Authors of the Ref. [33] analyzed the electronic transport in such ZMIs, and Ref. [34] has demonstrated experimental data confirming the presence of ZMI 5-8-5 D in graphene. ZMIs 5-8-5 A1 and 5-8-5 A3 were previously mentioned in Ref. [35] as interfaces between graphene and boron nitride crystals. In the same work [35], the stages of the formation of such interfaces. In our work, these interfaces are ZMIs between two graphene crystals.

An analysis of the atomic densities in ZMIs (Fig. 6) obviously shows that they are lower than the atomic density in defect-free graphene. However, there is no direct correlation between the density of atoms and the energy of the interface; therefore, the elastic field caused by the defect structure of the interface can be viewed as an important reason for the increase in the energy of ZMIs. A decrease in the atomic density in the ZMI region can increase the diffusion coefficient. The presence of such an effect may turn out to be essential for the modification of graphene with ZMIs with foreign atoms $[36,37]$. We plan to investigate the diffusion along and across ZMIs in the near future.

The linear energies of the low-energy ZMIs found with the help of two approaches: atomistic and mesoscopic, although differ numerically, they coincide in order of magnitude. The latter circumstance testifies to the prospects of using the mesoscopic approach. The observed differences, on the one hand, can be due to the fact that the analytical calculations of the ZMI energy were carried out within the framework of the linear theory of elasticity, which does not give accurate results near disclination cores. On the other hand, the energy mismatch can be caused by the imperfection of the chosen potential of interatomic interaction in the MD simulation. The potential used has proven itself to work well in simulating defect-free graphene, while the interfaces are composed almost entirely of defective carbon rings. In the future, we plan to use a numerical method based on the density functional theory (DFT). This will help to refine the results received for linear energies and structures, as well as simulate the physical properties of ZMIs in graphene.

MD modeling demonstrates that, low-energy ZMIs have linear energies not exceeding $\sim 0.6-0.8 \mathrm{eV} / \AA$, these values can be compared, for example, with the energy of the favorite grain boundary with a misorientation angle of $21.8^{\circ}$ in graphene $\sim 0.4 \mathrm{eV} / \AA$ [20].

\section{Summary and conclusions}

Using the atomistic and mesoscopic approaches, a set of zero misorientation interfaces (ZMIs) was simulated in graphene: the energies of ZMIs were found, the atomic densities of ZMIs were determined, and the distributions of the strain field components near the ZMIs were plotted.

It is shown that the mesoscopic approach, operating with the properties of disclinations in the elastic continuum, makes it possible, based on analytical expressions, to find the distributions of the elastic fields of interfaces and to estimate the energy of interfaces in graphene.

Acknowledgements. The support of RSF grant 19-19-00617 is acknowledged.

\section{References}

1. Z. S. Wu, W. Ren, L. Gao, J. Zhao, Z. Chen, B. Liu, D. Tang, B. Yu, C. Jiang, H. M. Cheng. ACS nano. 3 (2), 411 (2009). Crossref

2. A.A. Balandin, S. Ghosh, W. Bao, I. Calizo, D. Teweldebrhan, F. Miao, C. N. Lau. Nano Lett. 8 (3), 902 (2008). $\underline{\text { Crossref }}$

3. C. Lee, X. Wei, J. W. Kysar, J. Hone. Science. 321 (5887), 385 (2008). Crossref

4. K.S. Novoselov, A. K. Geim, S. V. Morozov, D. Jiang, Y. Zhang, S. V. Dubonos, I. V. Grigorieva, A. A. Firsov. Science. 306 (5696), 666 (2004). Crossref

5. F. Banhart, J. Kotakoski, A. V. Krasheninnikov. ACS nano. 5 (1), 26 (2011). Crossref

6. A.E. Romanov, M.A. Rozhkov, A.L. Kolesnikova. Lett. Mater. 8 (4), 384 (2018). Crossref

7. A. Bagri, S. P. Kim, R. S. Ruoff, V. B. Shenoy. Nano Lett. 11 (9), 3917 (2011). $\underline{\text { Crossref }}$

8. J. Lahiri, Y. Lin, P. Bozkurt, I. I. Oleynik, M. Batzill. Nature Nanotechnology. 5 (5), 326 (2010). Crossref

9. I. A. Ovid'ko. Rev. Adv. Mater. Sci. 34 (1), 1 (2013).

10. A. Luican-Mayer, J.E. Barrios-Vargas, J.T. Falkenberg, G. Autès, A.W. Cummings, D. Soriano, G. Li, M. Brandbyge, O. V. Yazyev, S. Roche, E. Y. Andrei. 2D Materials. 3 (3), 031005 (2016). Crossref

11. Z.X. Xie, Y. Zhang, L.F. Zhang, D. Y. Fan. Carbon. 113, 292 (2017). Crossref

12. R. Grantab, V. B. Shenoy, R. S. Ruoff. Science. 330 (6006), 946 (2010). Crossref

13. J. Stuart, A. B. Tutein, J. A. Harrison. J. Chem. Phys. 112 (14), 6472 (2000). Crossref

14. J. Tersoff. Phys. Rev. B. 37 (12), 6991 (1988). Crossref

15. K. Chenoweth, A.C. Van Duin, W. A. Goddard. J. Phys. Chem. A. 112 (5), 1040 (2008). ․ㅏossref 
16. A.P. Sgouros, G. Kalosakas, C. Galiotis, K. Papagelis. 2D Materials. 3 (2), 025033 (2016). Crossref

17. S. Cranford, M. J. Buehler. Modell. Simul. Mater. Sci. Eng. 19 (5), 054003 (2011). Crossref

18. H. Zhao, K. Min, N.R. Aluru. Nano Lett. 9 (8), 3012 (2009). Crossref

19. M.A. Rozhkov, A.L. Kolesnikova, I.S. Yasnikov, A.E. Romanov. Low Temp. Phys. 44 (9), 918 (2018). Crossref

20. A.L. Kolesnikova, M.A. Rozhkov, I. Hussainova, T. S. Orlova, I.S. Yasnikov, L. V. Zhigilei, A.E. Romanov. Rev. Adv. Mater. Sci. 52, 91 (2017).

21. T. Y. Ng, J. J. Yeo, Z.S. Liu. Carbon. 50 (13), 4887 (2012). Crossref

22. A.S. Kochnev, I.A. Ovid'ko, B.N. Semenov. Rev. Adv. Mater. Sci. 37, 105 (2014).

23. V. A. Kuzkin, A. M. Krivtsov. Doklady Physics. 56 (10), 527 (2011). Crossref

24. J. A. Baimova, L. Bo, S. V. Dmitriev, K. Zhou, A. A. Nazarov. EPL. 103 (4), 46001 (2013). Crossref

25. LAMMPS Molecular Dynamics Simulator Website

26. E. Polak, G. Ribiere. ESAIM: M2AN. 3 (R1), 35 (1969).

27. A. Stukowski. Modell. Simul. Mater. Sci. Eng. 20 (4), 045021 (2012). Crossref
28. A. E. Romanov, V. I. Vladimirov. In: Dislocations in Solids (ed. by F. R. N. Nabarro). North-Holland, Amsterdam (1992) $212 \mathrm{p}$.

29. A.E. Romanov, A.L. Kolesnikova, T.S. Orlova, I. Hussainova, V.E. Bougrov, R. Z. Valiev. Carbon. 81, 223 (2015). Crossref

30. I.S. Yasnikov, A. L. Kolesnikova, A.E. Romanov. Phys. Solid State. 58 (6), 1184 (2016). Crossref

31. MATLAB software package Website

32. M.A. Rozhkov, A.L. Kolesnikova, T.S. Orlova, L. V. Zhigilei, A.E. Romanov. Materials Physics and Mechanics. 29, 101 (2016).

33. C. J. Páez, A. L. C. Pereira, J. N. B. Rodrigues, N. M. R. Peres. Phys. Rev. B. 92 (4), 045426 (2015). $\underline{\text { Crossref }}$

34. J.H. Chen, G. Autès, N. Alem, F. Gargiulo, A. Gautam, M. Linck, C. Kisielowski, O. V. Yazyev, S. G. Louie, A. Zettl. Phys. Rev. B. 89 (12), 121407 (2014). Crossref

35. N. Ding, X. Chen, C. M. L. Wu. Scientific Reports. 6 (1), 1 (2016). Crossref

36. P. O. Lehtinen, A. S. Foster, A. Ayuela, A. Krasheninnikov, K. Nordlund, R. M. Nieminen. Phys. Rev. Lett. 91 (1), 017202 (2003). Crossref

37. X. Fan, W.T. Zheng, J.L. Kuo. ACS Applied Materials \& Interfaces. 4 (5), 2432 (2012). $\underline{\text { Crossref }}$ 